\title{
The effects of using variable lengths for degraded signal acquisition in GPS receivers
}

\author{
Arif Hussain ${ }^{1}$, Hina Magsi ${ }^{2}$, Arslan Ahmed ${ }^{3}$, Hadi Hussain ${ }^{4}$, Zahid Hussain Khand ${ }^{5}$, Faheem Akhtar ${ }^{6}$ \\ ${ }^{1,2,3,4}$ GNSS and Space Weather Lab, Department of Electrical Engineering, Sukkur IBA University, Pakistan \\ ${ }^{5,6}$ Department of Computer Science, Sukkur IBA University, Pakistan
}

\begin{tabular}{|c|c|}
\hline Article Info & ABSTRACT \\
\hline Article history: & The signal acquisition in GPS receivers is the first and very crucial process that may \\
\hline Received Aug 24, 2020 & affect the overall performance of a navigation receiver. Acquisition program initiates \\
\hline Revised Dec 10, 2020 & earching operation on received navigation signals to detect and identify the visible \\
\hline Accepted Jan 13, 2021 & $\begin{array}{l}\text { satellites. However, signal acquisition becomes a very challenging task in a degraded } \\
\text { environment (i.e, dense urban) and the receiver may not be able to detect the satel- }\end{array}$ \\
\hline Kevn & $\begin{array}{l}\text { lites present in radio-vicinity, thus cannot estimate an accurate position solution. In } \\
\text { such environments satellite signals are attenuated and fluctuated due to fading in- }\end{array}$ \\
\hline & troduced by multipath and NLOS reception. To perform signal acquisition in such \\
\hline Acquisition & degraded environments, larger data accumulation can be effective in enhancing SNR, \\
\hline Data length & which tradeoff huge computational load, prolonged acquisition time and high cost of \\
\hline GPS & receiver. This paper highlights the effects of fading on satellite signal acquisition in \\
\hline Signal-to-noise ratio & GPS receiver through variable data lengths and SNR comparison, and then develops \\
\hline Software receiver & $\begin{array}{l}\text { a statistical relationship between satellite visibility and SNR. Furthermore it also ana- } \\
\text { lyzes/investigates the tradeoff between computation load and signal data length. }\end{array}$ \\
\hline
\end{tabular}

This is an open access article under the $\underline{C C B Y-S A}$ license.

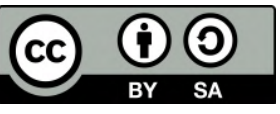

Corresponding Author:

Faheem Akhtar

Department of Computer Science

Sukkur IBA University

Sukkur, Sindh, Pakistan

Email: fahim.akhtar@iba-suk.edu.pk

\section{INTRODUCTION}

Global positioning system (GPS) is a satellite-based navigation system that provide positioning and localization service to both military and civilian applications [1-3] across the globe. This navigation system utilizes direct sequence code division multiple access (DS-CDMA) technique for signal transmission and it operates on L1 (1575.42 MHz) and L2 (1227.6 MHz) frequency bands. The GPS signals at L1 band, mainly utilized in civilian applications use pseudorandom noise (PRN) codes or coarse acquisition (C/A) to spread 50 $\mathrm{Hz}$ navigation message into wider spectrum. The C/A code has $1.023 \mathrm{MHz}$ chipping rate containing 1023 chips over period of 1 millisecond and each satellite has distinct C/A code that is used to identify the satellites. The precise $\mathrm{P}(\mathrm{Y})$ code is used to spread L2 band signals having chipping rate of $10.23 \mathrm{MHz}$ [4-6]. It is utilized in military applications because it can offer a higher tolerance to jamming and spoofing than C/A code. In the end, signals are modulated by binary phase shift keying (BPSK) and broadcasted at L band frequencies. The GPS signal structure is given in Figure 1.

The receivers acquire and process the signals broadcasted by satellites that revolve in medium earth orbit (MEO) at altitude of 20,200 KM to provide users with precise positioning, navigation and timing (PNT) 
information [5, 7]. Compared to conventional hardware based receiver, GPS software receiver performs wide range of signal operations in software based utility and can be easily reconfigured by updating algorithm deployed without making any changes in hardware. It performs digital base band signal processing to obtain navigation solutions capable processing real and complex signals (In phase and Quadrature components) at numerous sampling rates. The software receiver offer reasonable flexibility and are more robust compared to hardware counterpart. It can process real and complex signals (In phase and Quadrature components) at numerous sampling rates [8-10].

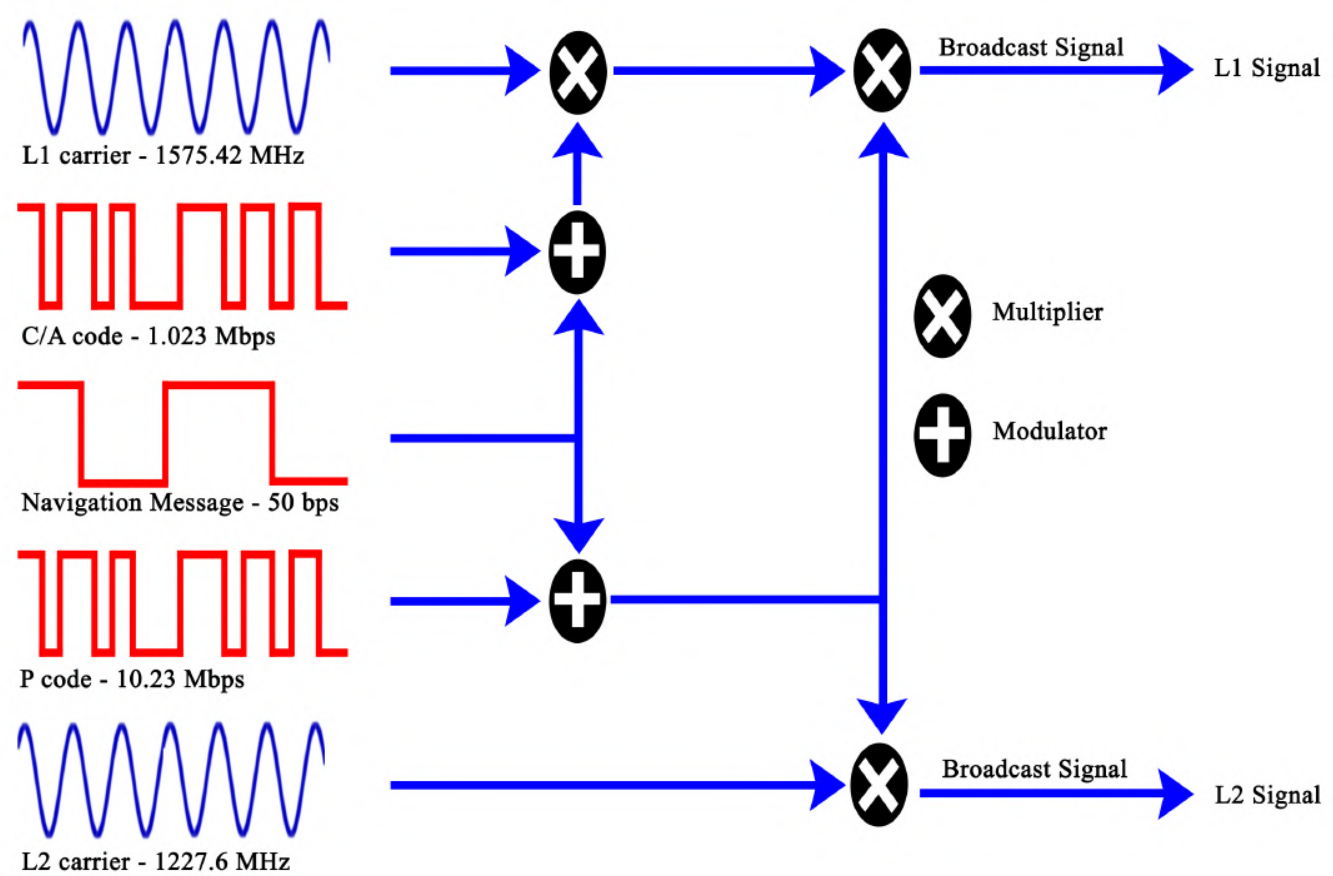

Figure 1. Fundamental structure of GPS signal; codes and data rates

A GPS receiver consist of radio frequency (RF) front end and software module, the RF front end pre-process the analog signal in raw form to make it compatible for software based processing. It performs frequency down conversion, amplification and digitization of a received signal as shown Figure 2 [11, 12]. The digitized signal at down converted frequency is passed to software part that consist of acquisition, tracking and navigation data extraction modules. The acquisition program performs a searching operation on block of data (received signal after digitization) to find out the signals from visible satellites. Once signal is acquired such as its presence is detected, the carrier frequency and code phase of signal is obtained and passed to tracking module. The code phase represents the point in current data block where C/A code begins and carrier frequency denotes the actual frequency of signal because Doppler shift can change the frequency. In literature, there exists several methods that are used for satellite signal acquisition such as serial search acquisition, parallel code phase search acquisition (FFT-based circular correlation) [13, 14], single side band (SSB) circular correlation, delay and multiply approach and zero padding (ZP) $[15,16]$ some methods are capable to acquire weak signals but are slower, while fast methods such as ZP may not be able to acquire degraded signals [5, 17].

The GPS signal acquisition can be influenced by delay imposed due to irregularities in Ionosphere and troposphere, receiver and satellite clock errors and multipath and environmental effects [18-22]. Therefore, to acquire a degraded GPS signal prolonged data lengths are taken to increase the input SNR but at the expense of exhaustive search and slower acquisition speed. In this paper, we are analyzing/investigating the effects of using variable data lengths for satellite signal acquisition. This analysis includes comparison of input SNR, computational load, and acquisition speed at different data lengths using FFT-based circular correlation and zero padding method. 


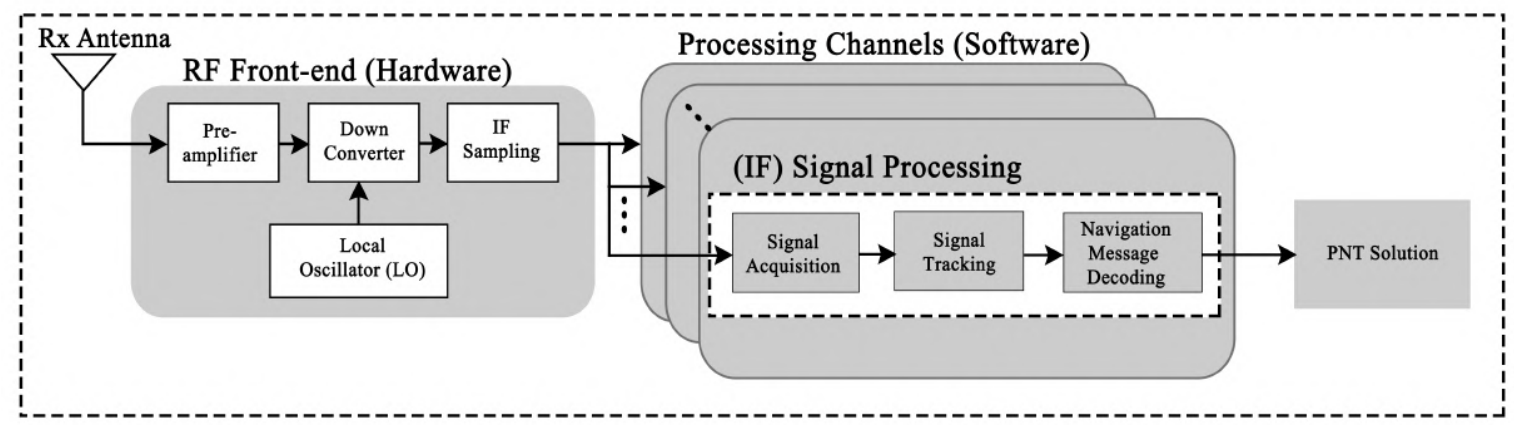

Figure 2. Block diagram of GPS receiver

\section{GPS SIGNAL STRUCTURE AND ACQUISITION}

The GPS satellites operate on L-band frequencies and transmit two distinct signals at L1 and L2 frequencies [23-25], in this paper we focus on L1 band modulated by civilian code C/A. The GPS is CDMA system so the received signal $S(t)$ is composite signal of $N$ visible satellites and it can be modelled as (1),

$$
S(t)=\sum_{k=1}^{N} s_{L 1}^{k}(t)
$$

where, $s_{L 1}^{k}(t)$ represents the actual signal model of given satellite (i.e., k) and can be given as (2).

$$
s_{L 1}(t)=M_{c} C(t) N(t) \cos \left(2 \pi\left(f_{L 1}+f_{d}\right) t\right)+M_{p} P(t) N(t) \sin \left(2 \pi\left(f_{L 1}+f_{d}\right) t\right)
$$

Where:

$M_{c}$ : is the magnitude of $\mathrm{C} / \mathrm{A}$ code

$C(t)$ : is the C/A code of the satellite

$N(t)$ : is the navigation data bits having values \pm 1

$f_{L 1}$ : is the $L 1$ carrier frequency and $f_{d}$ : is the Doppler shift

$M_{p}$ : is the magnitude precise code or $P(Y)$

$P(t)$ : is the precise code or $P(Y)$

The RF front end acquires incoming signal and after some signal conditioning it down converts it to an intermediate frequency $\left(f_{I F}\right)$. In this paper the signal is down-converted at $1.42 \mathrm{MHz}$ and mathematically it is given as (3).

$$
s_{I F}(t)=M_{c} C(t) N(t) \cos \left(2 \pi\left(f_{I F}+f_{d}\right) t\right)+M_{p} P(t) N(t) \sin \left(2 \pi\left(f_{I F}+f_{d}\right) t\right)
$$

The down-converted signal $\left(s_{I F}(t)\right)$ is then sampled and digitized by analog to digital converter (ADC) and passed through low pass filter (LPF) centered around C/A code. The LPF distorts $P(Y)$ code and it cannot be demodulated. Therefore, second part of (3) is modeled as error $(e(n))$ in the digitized signal.

$$
s(n)=M_{c} C(n) N(n) \cos \left(2 \pi\left(f_{I F}+f_{d}\right) n T_{s}\right)+e(n)
$$

where, $T_{s}$ is the sampling time $\left(T_{s}=1 / f_{s}\right)$ and $f_{s}$ is the sampling frequency and here $f_{s}=5 \mathrm{MHz}$. In general, the acquisition program initially wipes off the carrier signal and then performs correlation operation between received signal $s(n)$ and locally generated replica of transmitted C/A [26, 5] or PRN code $s_{l o}(n)$. If the correlation peak cross certain threshold such as $s(n)$ and $s_{l o}(n)$ match at some point then acquisition parameters (code phase and Doppler shift) are obtained and satellite is said to be acquired. The time domain and correlation $y(n)$ between finite sequence of received signal and locally generated C/A code can be given as (5). 


$$
y(n)=\sum_{i=1}^{N-1} s(i) s_{l o}(i+n)
$$

In order to perform FFT-based circular correlation, $y(n)$ is transformed into frequency domain and can be given as (6).

$$
Y(k)=\sum_{n=0}^{N-1} y(n) W_{N}^{n k}=\sum_{n=0}^{N-1} \sum_{i=1}^{N-1} s(i) s_{l o}(i+n) W_{N}^{n k}
$$

where, $W_{N}^{n k}=e^{-j 2 \pi k n / N}$ is the twiddle factor. In order to simplify, in (6) can be re-written as (7).

$$
Y(k)=S(k) S_{l o}^{*}(k)
$$

where, $S_{l o}^{*}(k)$ is the complex conjugate of $S_{l o}(k)$. Furthermore, correlation results in time domain $(y(n))$ can be obtained by taking inverse DFT. Once correlation is performed then correlation peak $\left(P_{N, \max }\right)$ is found by (8) and If the correlation peak in $y(n)$ cross certain threshold $(\gamma)$ then signal is said to be acquired otherwise frequency of local oscillator is changed and acquisition steps are repeated. The acquisition is declared only if $\left|P_{N, \max }\right|^{2}>\gamma$.

$$
\begin{aligned}
& P_{N, \max }=\max |y(n)|_{N} \\
& \gamma=\left(-2 \sigma_{n}^{2} \ln \left(P_{f a}\right)\right)^{1 / 2}
\end{aligned}
$$

where, $\gamma$ is the acquisition threshold, $\sigma_{n}^{2}$ is the noise variance and $P_{f a}$ is the probability of false alarm. The FFT-based method takes block of data, locally generated signal and performs correlation in frequency domain. The functional block diagram of FFT-based circular correlation is given in Figure 3.

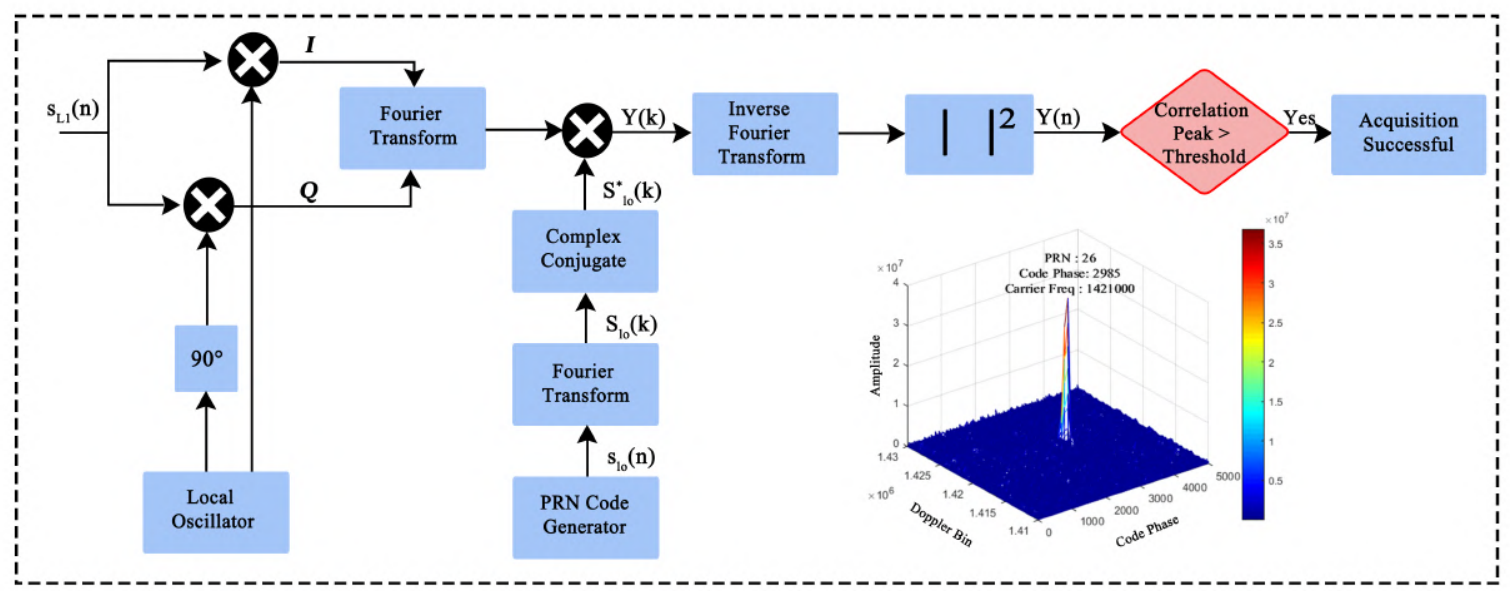

Figure 3. Block diagram of FFT-based circular correlation

The zero padding (ZP) method is similar to FFT-based method it also performs correlation in frequency domain. But ZP method utilizes divide and conquer strategy to speed up the acquisition process. It splits the data block of incoming signal and locally generated signal containing $N_{d}$ data samples into multiple blocks of smaller size $N_{d p b}$ and performs partial correlation. The block diagram of zero padding method is given in Figure 4. 

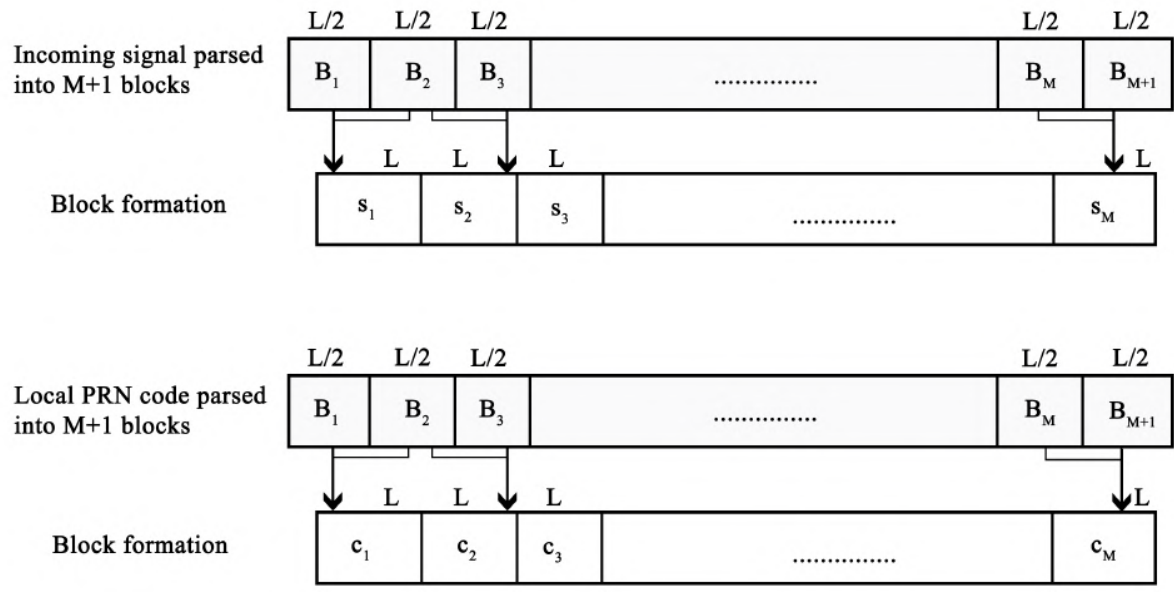

Figure 4. Block diagram of zero padding method [24]

\section{RESEARCH METHOD}

In this work/study the actual signal (real data) is used in acquisition process and three test signals are generated by adding $5 \mathrm{~dB}, 10 \mathrm{~dB}$ and $15 \mathrm{~dB}$ fading by introducing Gaussian noise into real data. The GPS signal is down converted at $1.42 \mathrm{MHz}$ and sampled at $5 \mathrm{MHz}$ therefore signal duration of 1 millisecond (msec) contains 5000 samples. The acquisition is performed on tests signals using both FFT-based circular correlation and zero padding method on $1 \mathrm{msec}$ and then non-coherent integration is performed up to $20 \mathrm{msec}$ of data. The Doppler frequency search range is $\pm 10 \mathrm{kHZ}$ with the step size of $1 \mathrm{KHz}$. In both algorithms code phase and doppler shift are observed to be exactly same but the magnitude of correlation peak is lesser in ZP method.

\subsection{Non-coherent integration}

The signal processing operations seek to boost SNR to improve the performance of system it can be done by adding (integrating) multiple data blocks together. In non-coherent integration data blocks are added non coherently such as each data block is processed separately and then results are added together.

$$
y_{N}(n)=\sum_{i=1}^{L}\left|y_{i}(n)\right|
$$

Where, $y_{N}(n)$ is the acquisition results after integrating $\mathrm{L}$ data blocks and $i=2,3, \ldots, \mathrm{L}, y_{i}(n)$ is the acquisition result of $i^{t h}$ data block and $L$ is intregation length. The non coherent integration gain $G_{n c}$ represents increment in the SNR when data blocks are added together and it is given by (11)

$$
G_{n c}^{i}=S N R^{i} / S N R^{i-1}
$$

where, $G_{n c}^{i}$ is the Gain at $i^{t h}$ integration and $S N R^{i}$ is the SNR at $i^{t h}$ integration.

\section{RESULTS AND DISCUSSION}

The Figure 5(a) and (b) shows the SNR results of signals acquired using FFT-based and ZP method respectively. The acquisition is performed on 3 test signals and it is observed that in both methods SNR is improved as the integration length increases. However, the FFT-based seems to be more robust as compared to ZP method because at same integration level it has higher value of SNR. On average there is $10 \mathrm{~dB}$ difference in the SNR of both methods on given integration time. This study concludes that ZP may not be able to acquire the signals in degraded environment even on larger integration length on the other hand FFT-based method is more robust and can acquire the weak signals comparatively at low integration lengths. 

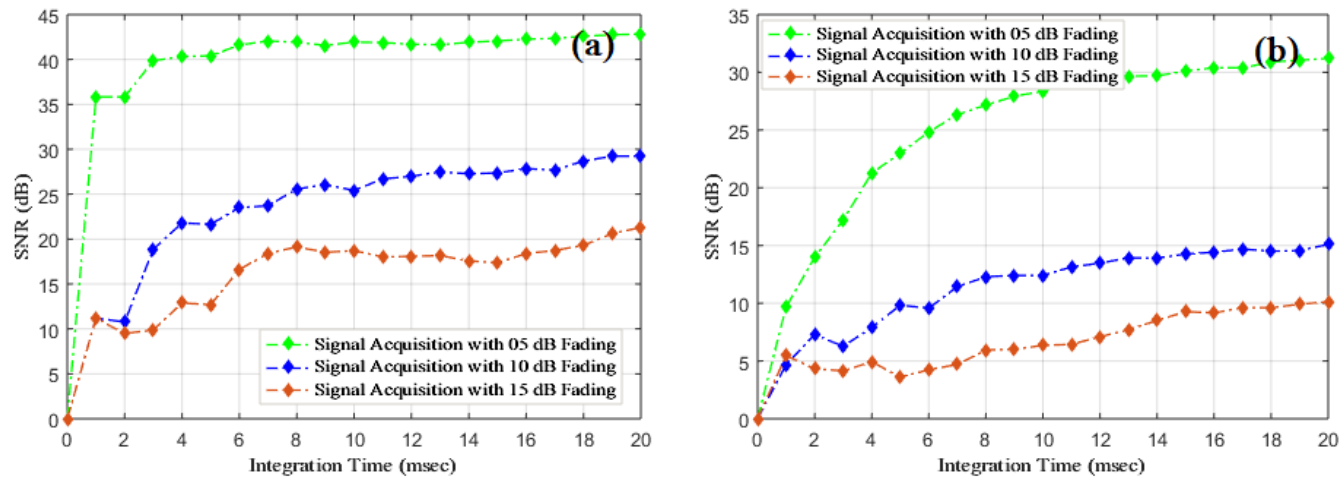

Figure 5. SNR result, (a) SNR of signals acquired by FFT-based method (b) SNR of signals acquired by ZP based method

\subsection{Acquisition results}

The acquisition is performed on three test signals and these test signals represents the fading levels that can occur in signals due to space weather effects, multipath effects and environmental effects. This analysis compares the acquisition performance of both methods on each test signal as well as on fading levels. It is observed that on each test signal code phase and carrier frequency obtained from both are exactly same but magnitude of correlation peak and acquisition time varies. For each test signal two types results are presented, initially summarized results are presented that depicts the correlation peak and acquisition threshold on integration interval and then detailed correlation results on certain integration interval presented. Both of the results depict a point where the satellite is acquired such as correlation peak cross the acquisition threshold. The acquisition is declared only if $\left|P_{N, \max }\right|^{2}>\gamma$ and threshold is obtained from (9) by keeping $P_{f a}=10^{-3}$. In order to avoid false acquisition declarations the threshold level is scaled up. The scaling factor for FFT-based method is set to 5 (because of robustness) and 2.50 for ZP method.

\subsection{First test signal}

The summarized acquisition results of first test signal ( $5 \mathrm{~dB}$ fading) over the integration interval of 20 msec are given in Figure 6(a) and (b). The vertical blue dotted line indicates the point (integration time) where acquisition is declared. FFT-based circular correlation is robust method and the acquisition is declared in the 1st msec of data because it has higher value of SNR as shown in Figure 5. However, it takes $3 \mathrm{msec}$ integration for same signal in ZP method to declare acquisition. Furthermore, it is observed that the magnitude correlation peak is much higher in FFT-based as compared to ZP method, for instance at $20^{\text {th }}$ msec correlation peak in FFT-based method is approximately 20 times the peak in ZP method.
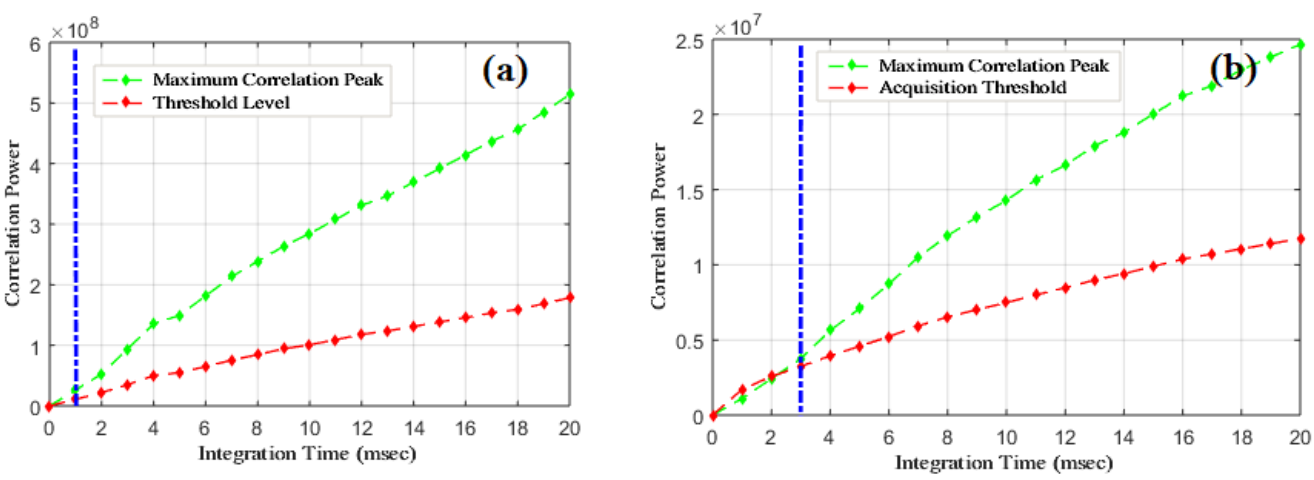

Figure 6. Signal acquisition at $5 \mathrm{~dB}$ fading, (a) using FFT-based method (b) using Zero Padding method

The correlation results over different data lengths (integration time) are given in Figures 7 and 8. The $\mathrm{x}$ component of DataTip represents the code phase and y component indicates the peak magnitude. The 
Doppler shift is $1 \mathrm{KHz}$ is observed therefore carrier frequency becomes $1.421 \mathrm{MHz}(1.42 \mathrm{MHz}+1 \mathrm{KHz})$. The results indicate that in both methods once the satellite is acquired the code phase and Doppler shift are same while the magnitude level is less in ZP method. It seems that ZP method has lower acquisition speed and high computational load because apparently it is taking more time to declare acquisition as compared to FFT-based method. However, computational analysis shows that ZP takes lower number of multiplications and additions to perform acquisition because of its divided approach.


Figure 7. Signal acquisition in GPS receiver using FFT-based circular correlation method at 5 dB fading
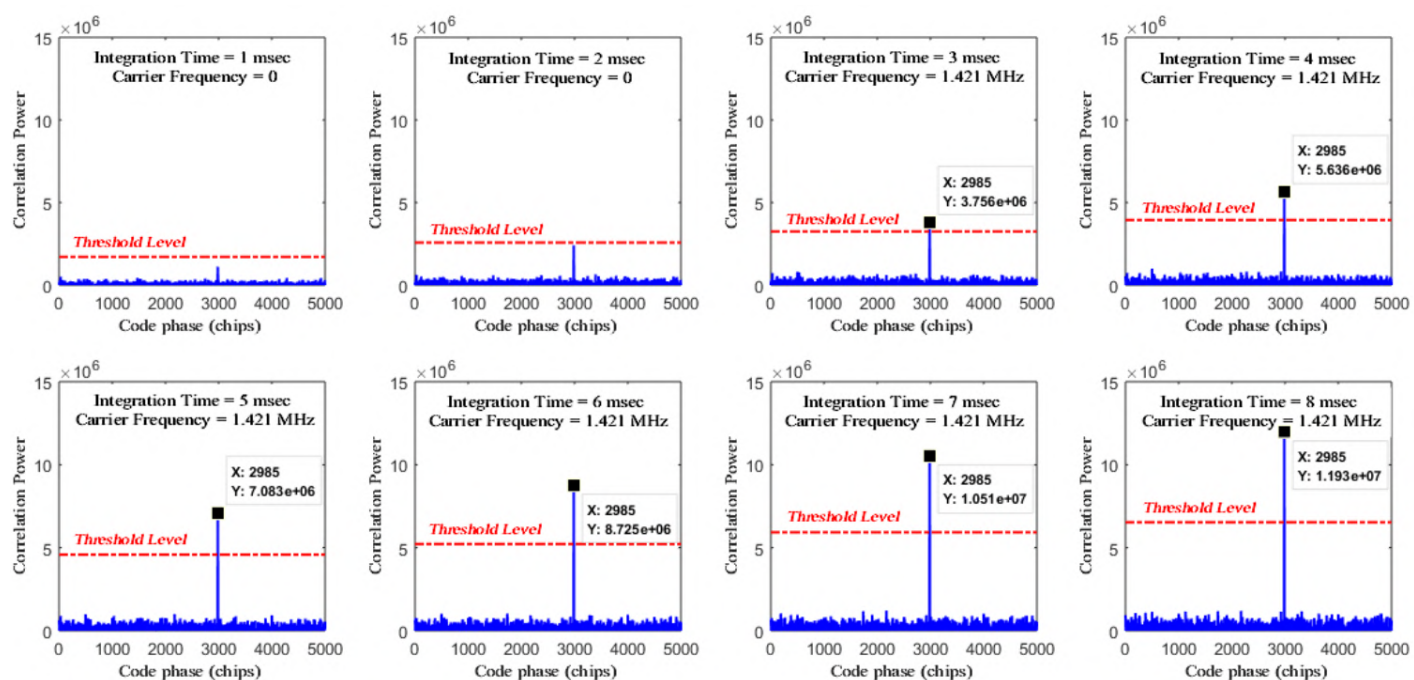

Figure 8. Signal acquisition in GPS software receivers using zero padding method at $5 \mathrm{~dB}$ fading

\subsection{Second test signal}

As the fading increases it becomes problematic to acquire the GPS signal, as it reduces the SNR and requires exhaustive search for signal detection. On second test signal (10 dB fading) FFT-based method declares the acquisition in the 4th msec while ZP method declares the acquisition at 8th msec as shown in Figure 9(a) and (b). The detailed correlation results over different data lengths (integration time)are given in Figures 10 and 11. 

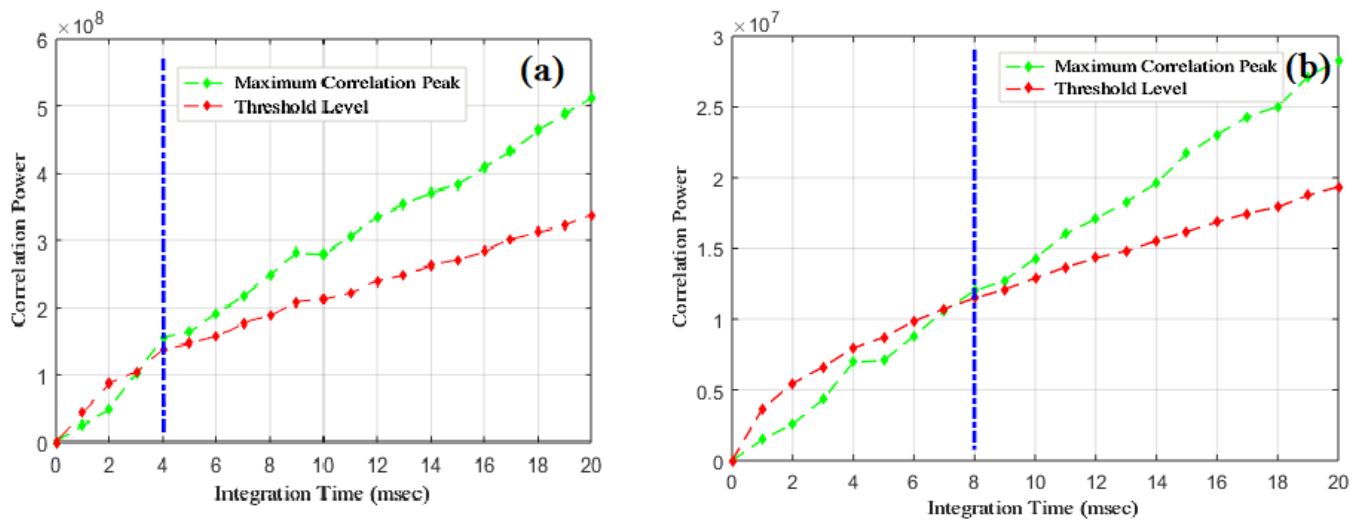

Figure 9. Signal acquisition at $10 \mathrm{~dB}$ fading, (a) using FFT-based method (b) using Zero Padding method
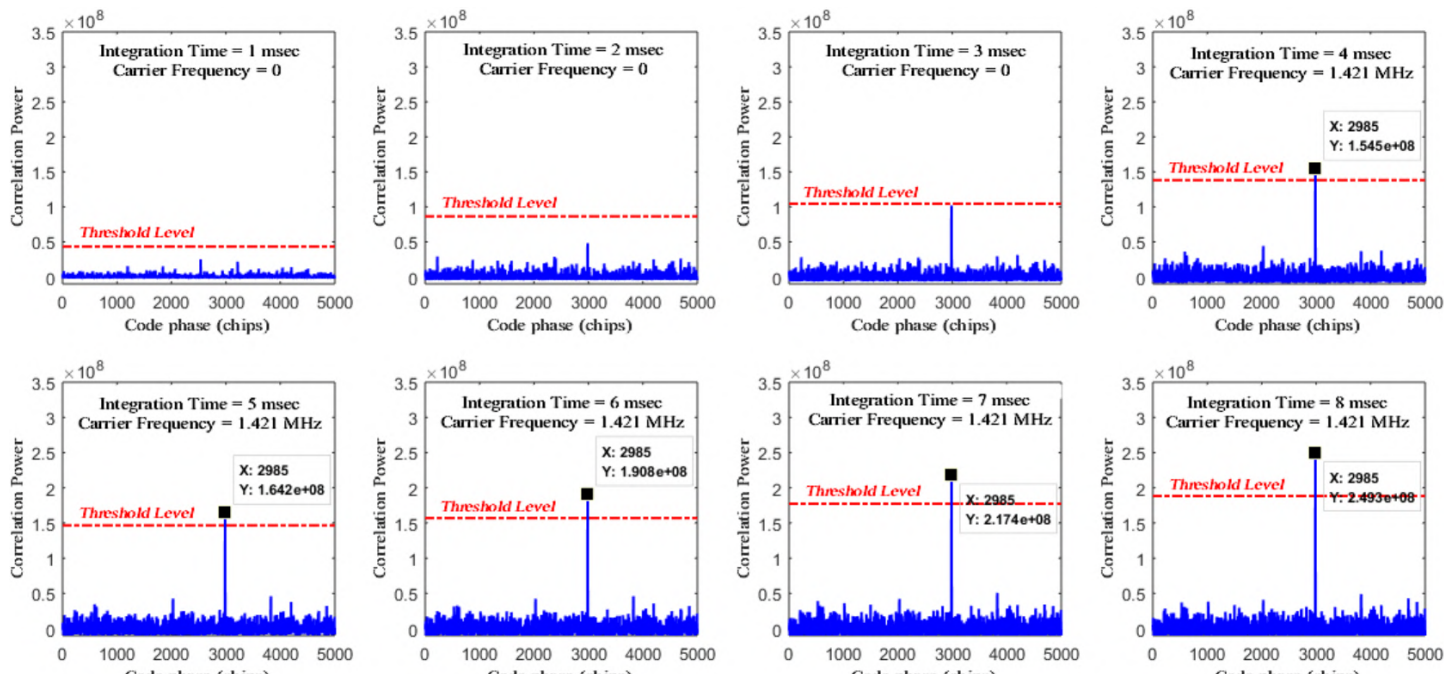

Figure 10. Signal acquisition in GPS receiver using FFT-based circular correlation method at 10 dB fading
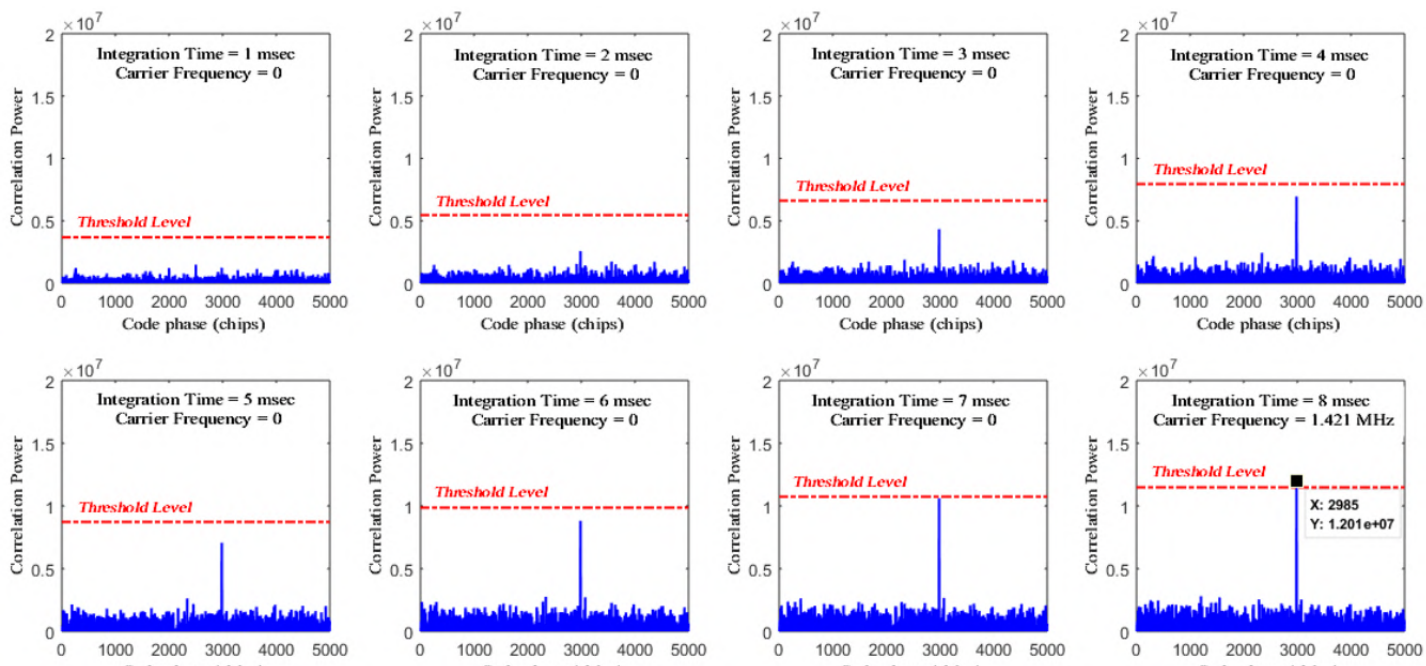

Code phase (chips)

Code phase (chips)

Code phase (chips)

Figure 11. Signal acquisition in GPS software receivers using Zero Padding method at $10 \mathrm{~dB}$ fading 


\subsection{Third test signal}

Sometimes due to ionospheric irregularities and severe multipath effects, the strength of GPS signal is very much deteriorated and may cause acquisition failure. It can introduce 15 to $20 \mathrm{~dB}$ of fading so, to analyze the performance of receiver in such conditions the acquisition is performed on a test signal having $15 \mathrm{~dB}$ of fading as shown in Figure 12(a) and (b). It is observed that in FFT-based method signal is acquired at $7^{\text {th }}$ integration point while in ZP method acquisition is declared at $15^{\text {th }}$ msec.

In general, we can say that the time to acquire the satellite increases as the fading level increases, however it is acquisition speed is very critical parameter in navigation receivers because the starting time of receiver depends upon. The computational analysis shows that ZP method is much faster than FFT-based method. The detailed correlation results of third test signal are not given because it follows the same pattern as of other test signals.
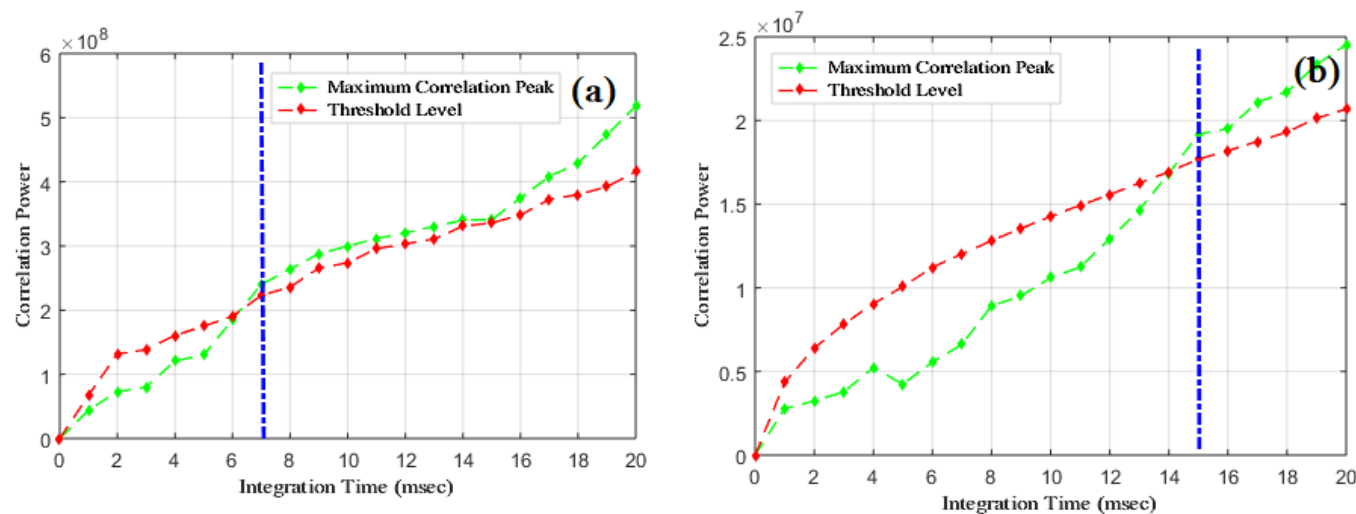

Figure 12. Signal acquisition at $15 \mathrm{~dB}$ fading, (a) using FFT-based method (b) using zero padding method

\section{DETECTION PERFORMANCE}

The output of correlator contains both signal $\hat{S}(k)$ and noise $\hat{n}(k)$ components.

$$
Y(k)=\hat{S}(k)+\hat{n}(k)
$$

In the absence of signal component such as when only noise signal is present then according to [17] probability density of noise envelope $p(r)$ is given by (13) and the probability of false alarm $P_{f a}$ is obtained by integration of (13).

$$
\begin{gathered}
p(r)=r / \sigma_{n}^{2} e^{-\left(\gamma^{2} / 2 \sigma_{n}^{2}\right)} \\
P_{f a}=\int_{\gamma}^{\infty} p(r) d r=e^{-\left(\gamma^{2} / 2 \sigma_{n}^{2}\right)}
\end{gathered}
$$

Now if the signal component is also present having amplitude $A$ then probability density of noise envelope $p(r)$ will follow Rician distribution [18] and given as (15).

$$
p(r)=\frac{r}{\sigma_{n}^{2}} \exp \left(-\frac{\gamma^{2}+A^{2}}{2 \sigma_{n}^{2}}\right) I_{o}\left(\frac{A r}{2 \sigma_{n}^{2}}\right)
$$

where, $A^{2}$ is the $\left|P_{N, \max }\right|^{2}$ and $I_{o}(Z)$ is the zero order modified Bessel function of the first kind. The probability of detection $\left(P_{d}\right)$ is the probability that signal will be acquired such as $r>\gamma$ is given by (16).

$$
P_{d}=\int_{\gamma}^{\infty} \frac{r}{\sigma_{n}^{2}} \exp \left(-\frac{\gamma^{2}+A^{2}}{2 \sigma_{n}^{2}}\right) I_{o}\left(\frac{A r}{2 \sigma_{n}^{2}}\right) d r
$$

The series approximation of detection probability [19] is given by (17). 


$$
P_{d}=0.5\left[1-\operatorname{erf}\left(\frac{\gamma-A}{\sqrt{2 \sigma_{n}^{2}}}\right)\right]+\left\{\frac{\exp \left[-(\gamma-A)^{2} / \sigma_{n}^{2}\right]}{2 \sqrt{2 \pi}\left(A / \sqrt{\left.\sigma_{n}^{2}\right)}\right.}\right\} \times\left\{1-\frac{\gamma-A}{4 A}+\frac{1+\left[(\gamma-A)^{2} / \sigma_{n}^{2}\right]}{8 A / \sigma_{n}^{2}}\right\}
$$

Furthermore, $P_{d}$ can be expressed as a function of SNR with $P_{f a}$ as parameter from (15) $\gamma / 2 \sigma_{n}^{2}=$ $\ln \left(1 / 2 P_{f a}\right)$ and using power relationship.

$$
\frac{A}{\sqrt{\sigma_{n}^{2}}}=\frac{\text { SignalAmplitude }}{\text { rmsnoisevoltage }}=2\left(\frac{\text { SignalPower }}{\text { NoisePower }}\right)=\sqrt{2 S N R}
$$

The detection probability $\left(P_{d}\right)$ of both FFT-based and ZP method over the integration interval for all three test signals is given in Figure 13(a) and (b) respectively. The detection performance indicates that $P_{d}$ gets better as integration length increases however it is also affected by fading. Like SNR, the probability that a signal is detected (acquired) reduces as fading level increases. In FFT-based method, at any given data length such as $6^{\text {th }}$ msec, the $P_{d}$ of $2^{\text {nd }}$ test signal is approximately $10 \%$ less than the $1^{\text {st }}$ test signal, similarly $P_{d}$ of $3^{\text {rd }}$ test signal is around $30 \%$ less than $1^{\text {st }}$ test signal. In case of $3^{\text {rd }}$ test signal detection performance is improved by nearly $40 \%$ over the integration interval of $20 \mathrm{msec}$.
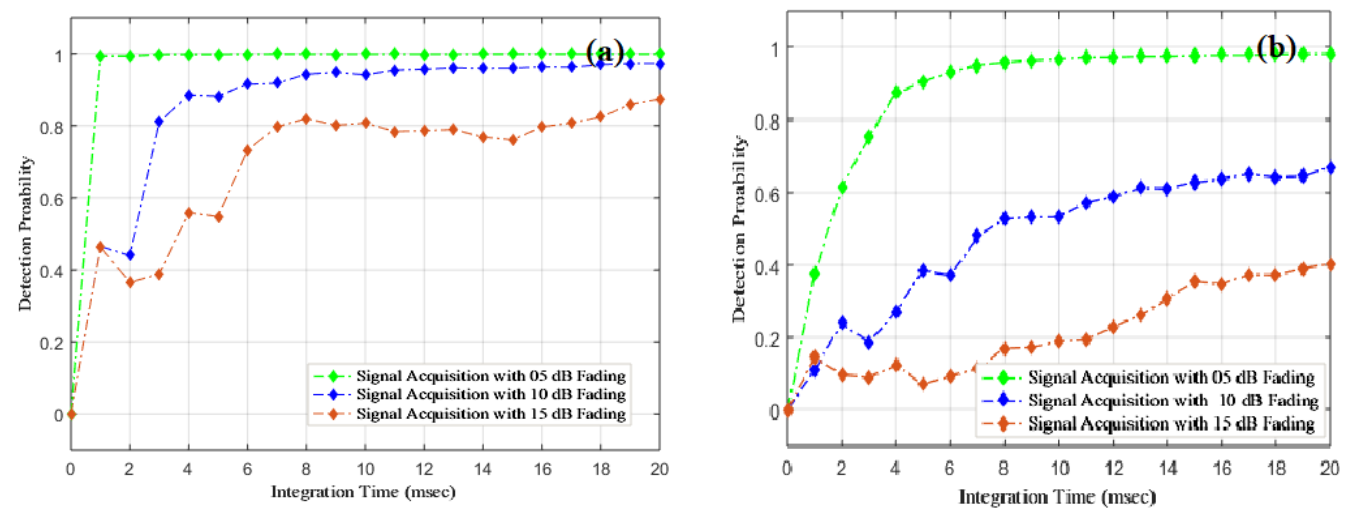

Figure 13. Results of third test signal, (a) Probability of detection using FFT-based method (b) Probability of detection ZP method

In ZP method, there is significant difference in the detection performance of test signals. For instance at $16^{\text {th }} \mathrm{msec}$, the $P_{d}$ of $2^{\text {nd }}$ test signal is approximately $35 \%$ less than the $1^{\text {st }}$ test signal, similarly $P_{d}$ of $3^{\text {rd }}$ test signal is around $60 \%$ less than $1^{\text {st }}$ test signal. Comparatively detection performance is significantly improved at low fading level (such as $1^{\text {st }}$ test signal) over the same integration interval. In case of $3^{\text {rd }}$ test signal detection performance is improved by nearly $30 \%$ over the integration interval of $20 \mathrm{msec}$. Comparative analysis of detection performance indicates that FFT-based method is more robust and can acquire signals in degraded environment. Its detection is less affected by fading introduced due to several effects as compared to ZP method.

\section{CONCLUSION}

This paper has discussed the effects of using variable lengths for signal acquisition in GPS software receivers. It is presented that detection performance and SNR can be improved by non-coherent integration of data lengths. The acquisition results showed that data length sets the trade-off among detection performance, SNR and acquisition speed. The study indicates that FFT-based method is much robust and can acquire degraded signals but it is slower than the ZP method.

\section{REFERENCES}

[1] E. G. N. S. S. A., "Gnss market report," PA, Annual report of the United Nations High Commissioner for Human Rights and reports of the Office of the High Commissioner and the Secretary-General, 2019, [Online]. Available: https://www.gsa.europa.eu/market/market-report. 
[2] J. Guo, X. Li, Z. Li, L. Hu, G. Yang, C. Zhao, D. Fairbairn, D. Watson, and M. Ge, "Multi-gnss precise point positioning for precision agriculture," Precision agriculture, vol. 19, no. 5, pp. 895-911, 2018.

[3] G. Xu and Y. Xu, "GPS: theory, algorithms and applications," Springer, 2016.

[4] K. Borre, D. M. Akos, N. Bertelsen, P. Rinder, and S. H. Jensen, "A Software-Defined GPS and Galileo Receiver: A Single-Frequency Approach," Springer Science and Business Media, 2007.

[5] J. B. Tsui, "GPS C/A Code Signal Structure, Chapter 5," John Wiley and Sons, pp. 68-101, 2005.

[6] C. Bruyninx, W. Aerts, and J. Legrand, "Gps, Data Acquisition and Analysis," Dordrecht: Springer Netherlands, pp. 420-431, 2011, doi: 10.1007/978-90-481-8702-7-137.

[7] A. Paul, K. S. Paul, and A. Das, "Impact of multiconstellation satellite signal reception on performance of satellite-based navigation under adverse ionospheric conditions," Radio Science, vol. 52, no. 3, pp. 416-427, 2017, doi: 10.1002/2016RS006076.

[8] F. Wu, K. Zhang, and A. Yasuda, "Gps signal acquisition and tracking using software gps receiver," in Eighth International Symposium on Signal Processing and Its Applications, 2005, pp. 843-846, doi:

[9] A. Alaqeeli, J. Starzyk, and F. Van Graas, "Real-time acquisition and tracking for gps receivers," in Proceedings of the 2003 International Symposium on Circuits and Systems, 2003, vol. 4, pp. IV-IV, doi: 10.1109/ISCAS.2003.1205933.

[10] P. Varzakas, "Average channel capacity for rayleigh fading spread spectrum mimo systems," International Journal of Communication Systems, vol. 19, no. 10, pp. 1081-1087, 2006.

[11] D. Manandhar, Y. Suh, and R. Shibasaki, "Gps signal acquisition and tracking-an approach towards development of software-based gps receiver," Technical Report of IEICE, 2004.

[12] J. Yin, R. Tiwari, and M. Johnston, "Low-cost dual polarized gps antenna for effective signal acquisition in multipath environment," in 2017 European Navigation Conference (ENC), 2017, pp. 359-365.

[13] X. Chen, J. Xu, T. Ye, and S.-c. LI, "Fft-based acquisition for weak gps signals," Microelectronics Comp., 2010.

[14] C. O'Driscoll and C. C. Murphy, "Performance analysis of an fft based fast acquisition gps receiver," in ION National Tech. Meeting, 2005, pp. 1014-1025.

[15] B. Chibout, C. Macabiau, A.-C. Escher, L. Ries, J.-L. Issler, S. Corazza, and M. O. Bousquet, "Comparison of acquisition techniques for gnss signal processing in geostationary orbit," Proceedings of the 2007 National Technical Meeting of The Institute of Navigation, 2007, pp. 637-649.

[16] D. M. Lin, "Comparison of acquisition methods for software GPS receiver," Proceedings of the 13th International Technical Meeting of the Satellite Division of The Institute of Navigation, 2000, pp. 2385-2390.

[17] D. M. Lin and J. B. Tsui, "Comparison of Acquisition Methods for Software GPS Receiver," Proceedings of the 13th International Technical Meeting of the Satellite Division of The Institute of Navigation, 2016, pp. 2385-2390.

[18] B. W. Parkinson and J. J. S. Jr., "Global Positioning System: Theory and Applications," Chapter 11. Wash- ington, DC: American Institute of Aeronautics and Astronautics, Inc, vol. 1, 1996.

[19] A. Ahmed, R. Tiwari, S. I. Ali, and G. Jaffer, "The effects of ionospheric irregularities on the navigational receivers and its mitigation," in International Conference for Emerging Technologies in Computing, Springer, 2018, pp. 87-97, doi: 10.1007/978-3-319-95450-9-7.

[20] P. Tregoning and C. Watson, "Atmospheric effects and spurious signals in gps analyses," Journal of Geophysical Research: Solid Earth, vol. 114, no. B9, 2009.

[21] J. Seo, T. Walter, and P. Enge, "Availability impact on gps aviation due to strong ionospheric scintillation," IEEE Transactions on Aerospace and Electronic Systems, vol. 47, no. 3, pp. 1963-1973, July 2011, doi: 10.1109/TAES.2011.5937276.

[22] F. K. Brunner and W. M. Welsch, "Effect of the troposphere on gps measurements," GPs World, vol. 4, p. $42,1993$.

[23] L. R. W. Mohinder S Grewal and A. P. Andrews, "Global positioning systems, inertial navigation and integration," John Wiley and Sons, 2007.

[24] A. Ahmed, "Scintillation on global navigation satellite signals and its mitigation," Ph.D. dissertation, Newcastle University, UK, 2015.

[25] F. Gao, "Performance analysis of long-time coherent integral acquisition algorithm for weak GNSS signals," IOP Conference Series: Earth and Environmental Science, vol. 428, p. 012002, jan 2020.

[26] A. Ahmed, R. Tiwari, H. Strangeways, and S. Boussakta, "Software-based receiver approach for acquiring GPS signals using block repetition method," 2014 7th ESA Workshop on Satellite Navigation Technologies and European Workshop on GNSS Signals and Signal Processing (NAVITEC), 2014, pp. 1-7. 